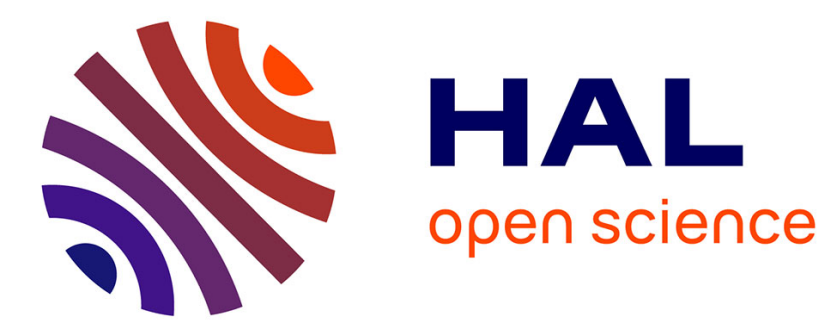

\title{
Periodic event-triggered control for nonlinear systems
} Romain Postoyan, Adolfo Anta, W.P.M.H. Heemels, Paulo Tabuada, Dragan

Nesic

\section{To cite this version:}

Romain Postoyan, Adolfo Anta, W.P.M.H. Heemels, Paulo Tabuada, Dragan Nesic. Periodic eventtriggered control for nonlinear systems. 52nd IEEE Conference on Decision and Control, CDC 2013, Dec 2013, Florence, Italy. pp.CD-ROM. hal-00875685

\section{HAL Id: hal-00875685 https://hal.science/hal-00875685}

Submitted on 15 Dec 2013

HAL is a multi-disciplinary open access archive for the deposit and dissemination of scientific research documents, whether they are published or not. The documents may come from teaching and research institutions in France or abroad, or from public or private research centers.
L'archive ouverte pluridisciplinaire HAL, est destinée au dépôt et à la diffusion de documents scientifiques de niveau recherche, publiés ou non, émanant des établissements d'enseignement et de recherche français ou étrangers, des laboratoires publics ou privés. 


\title{
Periodic event-triggered control for nonlinear systems
}

\author{
R. Postoyan, A. Anta, W.P.M.H. Heemels, P. Tabuada, D. Nešić
}

\begin{abstract}
Resources limitations of embedded systems and networked control systems have motivated the development of novel controller implementation paradigms in order to reduce the potential conservatism of traditional clock-driven setups. In this context periodic event-triggered control has recently been proposed. Based on a periodically evaluated and statedependent criterion, it is decided, at every sampling instant, whether the control input needs to be updated or not. In this paper we propose a method for the design of periodic event-triggered controllers for nonlinear systems. We follow an emulation-based approach as we start from a known (continuously evaluated) event-triggered controller and we provide a systematic technique to select the sampling period and to redesign the triggering condition to approximately maintain the guarantees ensured by the original controller. The method is illustrated on a physical system and we compare the obtained results with other available event-based implementations.
\end{abstract}

\section{INTRODUCTION}

Embedded systems and networked control systems require the development of novel controller implementation paradigms in order to cope with the ever tighten resources limitations which are typical for these systems. Indeed, the traditional setup, where data is periodically exchanged between the controller and the plant, may be no longer appropriate as it may lead to an excessive usage of the communication channel and of the CPU. In this context, eventtriggered control (ETC) has been proposed as an alternative to the periodic setup [3], [4]. The underlying idea is to adapt the transmissions and the controller execution to the current state of the plant. In ETC, the plant's states are continuously monitored and the loop is closed whenever a state-dependent criterion is satisfied. In that way, a transmission is triggered

R. Postoyan is with the Université de Lorraine, CRAN, UMR 7039 and the CNRS, CRAN, UMR 7039, France romain.postoyanduniv-lorraine.fr. $\mathrm{He}$ is partially supported by the European 7th Framework Network of Excellence "Highly-complex and networked control systems" (HYCON2) (grant agreement no. 257462).

A. Anta aantamegmail. com

W.P.M.H. Heemels is with the Department of Mechanical Engineering, Eindhoven University of Technology, Eindhoven, The Netherlands, m.heemels@tue.nl. He is supported by the Innovational Research Incentives Scheme under the VICI grant "Wireless control systems: A new frontier in automation" (No. 11382) awarded by NWO (The Netherlands Organization for Scientific Research) and STW (Dutch Science Foundation), and the European 7th Framework Network of Excellence "Highly-complex and networked control systems" (HYCON2) (grant agreement no. 257462).

P. Tabuada is with the Cyber-Physical Systems Laboratory of the Department of Electrical Engineering at the University of California, Los Angeles, CA, USA tabuada@ee. ucla . edu. His work was partially supported by the NSF award 1035916.

D. Nešić is with the Department of Electrical and Electronic Engineering, the University of Melbourne, Parkville, VIC 3010, Australia d.nesicdee.unimelb.edu.au. His work is supported by the Australian Research Council under the Discovery Grant and Future Fellowship schemes. only when an event occurs, otherwise the communication channel is not used for control. A number of studies have shown that ETC may be able to significantly reduce the amount of transmissions needed to stabilize a (nonlinear) system, see e.g. [3], [4], [10], [11], [15], [19], [23]. However, a downside of ETC is the requirement to continuously evaluate the triggering condition which is not conceivable when the implementation platform is digital. In practice, the triggering condition can only be verified periodically. In this case, it is decided at each sampling instant whether the control input needs to be updated, which turns the implementation to a periodic event-triggered control (PETC) system, a terminology introduced in [9], see also [3], [10]. The sampling of the triggering mechanism may deteriorate the performance of the ETC system compared to continuously evaluated ETC, in particular when the period cannot be made small enough due to platform limitations. In fact, one may aim for larger sampling periods in order to save (even more) computation and communication resources. There is therefore a strong need for systematic methods to design appropriate PETC strategies. Results regarding the robust stabilization of linear systems under PETC in terms of $\mathcal{L}_{2}$-gains are given in [8], [9].

The purpose of the paper is to propose PETC strategies for general nonlinear systems. We start from a continuously evaluated event-triggered controller which has been designed to ensure a desired specification for a nonlinear continuoustime plant. We then explain how to derive PETC policies which approximately maintain the properties ensured by the original controller. The approach uses the results on isochronous manifolds in [2] to analyze the evolution of the original event-triggering condition between two sampling instants. We show that the performance degradations induced by the sampling can be rendered as small as desired at the price of a more complex triggering condition. Compared to [8], [9], we address nonlinear systems and we emulate a (continuously evaluated) event-triggered controller while the results in [8], [9] start from a periodically sampled controller. The work in [18] investigates the effect of sample-and-hold devices on hybrid feedbacks and can thus be used to derive a PETC system based on a continuously evaluated ETC. Our work differs from [18] in the sense that we do not directly implement the triggering law from the (continuously evaluated) ETC system but we redesign it in order to ensure stronger properties. Furthermore, the presented approach is not only applicable for the purpose of stabilizing a compact attractor as in [8], [9], [18]: any ETC system can be turned to a PETC system as long as it satisfies the required conditions.

The results in this paper can be seen as an alternative to 
the self-triggered control (STC) method of [2]. In STC, the measurements are only collected at the triggering instants (and not periodically) and the next time at which the loop must be closed is then pre-computed, see e.g. [1], [2], [20], [21]. The techniques in [2] may need significant computation resources at each transmission instant to generate long inter-transmission intervals, while we will see that the derived PETC laws are typically simpler from the computational point of view compared to [2]. However, PETC may need to more often evaluate the triggering condition. On the other hand, PETC is expected to ensure stronger robustness properties than STC, as shown on an illustrative example, since the state of the plant is more frequently monitored in PETC than in STC.

Notation. Let $\mathbb{R}=(-\infty, \infty), \mathbb{R}_{\geq 0}=[0, \infty), \mathbb{Z}_{\geq 0}=$ $\{0,1,2, \ldots\}, \mathbb{Z}_{>0}=\{1,2, \ldots\}$. For $(x, y) \in \mathbb{R}^{n+m},(x, y)$ stands for $\left[x^{\mathrm{T}}, y^{\mathrm{T}}\right]^{\mathrm{T}}$. A function $\gamma: \mathbb{R}_{\geq 0} \rightarrow \mathbb{R}_{\geq 0}$ is of class $\mathcal{K}_{\infty}$ if it is continuous, zero at zero, strictly increasing and unbounded. The solution $z$ of a time-invariant dynamical system at time $t \geq 0$ starting with the initial condition $z(0)=$ $z_{0}$ will be denoted $z\left(t, z_{0}\right)$ or simply $z(t)$ when the initial state is clear from the context. For a piecewise continuous function $f: \mathbb{R} \rightarrow \mathbb{R}^{n}, f\left(t^{+}\right)$stands for $\lim _{s \rightarrow t, s>t} f(s)$ for $t \in \mathbb{R}$. The notation $\lfloor\cdot\rfloor$ denotes the floor function.

\section{Problem statement}

\section{A. Preliminaries on the event-triggered controller}

We consider a dynamical system of the form

$$
\dot{x}=f(x, u),
$$

where $x \in \mathbb{R}^{n_{x}}$ is the state which is assumed to be measured, $u \in \mathbb{R}^{n_{u}}$ is the control input. A control law of the form $u=k(x)$ has been designed in continuous time to satisfy a desired specification. We assume it to be static for the clarity of exposition only; our results also apply to dynamic statefeedback laws as it will be clear in the sequel (see Remark 1 below).

We consider a scenario where the controller is implemented on a digital platform. As a consequence, it is only at some time instants $t_{k}, k \in \mathbb{Z}_{\geq 0}$, that the measurements are sent to the controller, and that a new control input is computed and transmitted to the plant. When the input is held constant between updates, (1) becomes, for all $t \in\left[t_{k}, t_{k+1}\right.$ [

$$
\dot{x}(t)=f\left(x(t), k\left(x\left(t_{k}\right)\right)\right)=f(x(t), k(x(t)+e(t))),
$$

where $e$ represents the difference between the last transmitted value of the state to the controller and the current state

$$
e(t)=x\left(t_{k}\right)-x(t), \quad \forall t \in\left[t_{k}, t_{k+1}[.\right.
$$

We consider the sampling-induced error on the state $x$ but we could have equivalently chosen to introduce the error induced on the input, i.e. $k\left(x\left(t_{k}\right)\right)-k(x(t))$ (see Section VII.B in [19] for a discussion on this point). In conventional setups, the controller is typically updated in a periodic fashion, i.e. $t_{k+1}-t_{k}=h$ where $h>0$ is known as the sampling period. Event-triggered control, on the other hand, updates the control input whenever a state-dependent criterion is satisfied. The triggering condition can be defined using a function $\Gamma: \mathbb{R}^{2 n_{x}+n_{\chi}} \rightarrow \mathbb{R}$ which depends on the state $x$, the sampling-induced error $e$ and potentially on some additional variables $\chi \in \mathbb{R}^{n_{\chi}}$ which may be introduced by the user (see [15], [16] for instance). Without loss of generality, we assume that $\Gamma$ is negative after a jump and that the next transmission instant occurs as soon as $\Gamma$ becomes non-negative. Using an impulsive formulation as in [6], [9], the closed-loop system under an event-triggering strategy can be defined as

$$
\begin{aligned}
\dot{z} & =g(z) \quad \forall t \in\left[t_{k}, t_{k+1}[\right. \\
z\left(t_{k+1}^{+}\right) & =b\left(z\left(t_{k+1}\right)\right),
\end{aligned}
$$

for appropriate $g: \mathbb{R}^{n_{z}} \rightarrow \mathbb{R}^{n_{z}}$ and $b: \mathbb{R}^{n_{z}} \rightarrow \mathbb{R}^{n_{z}}$ where $z=(x, e, \chi) \in \mathbb{R}^{n_{z}}$ with $n_{z}=2 n_{x}+n_{\chi}$. We typically have $b(z)=(x, 0, c(x, e, \chi))$ where the continuous mapping $c$ defines the behaviour of the designed $\chi$-system at jumps. However, it has to be noted that the assumption that $e$ is reset to 0 after each jump is not needed; such a scenario occurs in decentralized setups, see e.g. [6], [12], [16], [22]. The jump instants are defined by the following rule

$$
t_{k+1}=t_{k}+\min \left\{t>0: \Gamma\left(z\left(t, b\left(z\left(t_{k}\right)\right)\right)\right) \geq 0\right\}, \quad t_{0}=0,
$$

where $z\left(t, z_{0}\right)$ is the solution to $\dot{z}=g(z)$ at time $t \geq 0$ starting at $z_{0}$. The function $\Gamma: \mathbb{R}^{n_{z}} \rightarrow \mathbb{R}$ in (5) is designed in such a way that updating the control input at time instants $t_{k}$ guarantees that for any solution $z$ to (4)-(5) the following holds

$$
\Gamma(z(t)) \leq 0 \quad \forall t>0,
$$

which ensures stability or required performance for the closed-loop. For instance, stabilizing event-triggered controllers are designed in [19] with a triggering law of the form of $\gamma(|e|) \geq \sigma \alpha(|x|)$ with $\gamma, \alpha \in \mathcal{K}_{\infty}$ and $\sigma \in(0,1)$. Hence, we obtain $\Gamma(x, e)=\gamma(|e|)-\sigma \alpha(|x|)$. Similarly, the conditions of the form $|e| \geq \rho$ where $\rho>0$ used in [3], [4], [13], [14] to practically stabilize the origin of nonlinear systems give $\Gamma(e)=|e|-\rho$. It is explained in [2] and in Section V of [16] how $\Gamma$ can be constructed for other types of event-triggering conditions.

We assume that the hybrid system (4)-(5) has leftcontinuous solutions which exist for all time and for every considered initial condition. In that way, to obtain a solution in the sense of Carathéodory, we flow the continuous dynamics until a jump occurs and then we flow again and so on.

We make the following assumption on system (4)-(5).

Assumption 1: The jumps induced by $\Gamma$ on the eventtriggered system (4)-(5) are spaced at least by $T>0$ units of time, i.e.

$$
T:=\inf _{z_{0} \in \Omega}\left\{t>0: \Gamma\left(z\left(t, b\left(z_{0}\right)\right)\right) \geq 0\right\}>0,
$$

where $z$ is solution to $\dot{z}=g(z)$ and $\Omega \subseteq \mathbb{R}^{n_{z}}$ is a compact 
forward invariant ${ }^{1}$ set for system (4)-(5).

Assumption 1 is reasonable as the event-triggering condition clearly needs to ensure the existence of a uniform minimum amount of time between two transmissions over a given operating set $\Omega$ in order to satisfy the inherent hardware constraints. This condition is verified by most available event-triggering schemes in the literature. The set $\Omega$ can be determined using the level set of some Lyapunov function when investigating stabilization problems for example.

Remark 1: The forthcoming analysis applies to system (4)-(5). We therefore see that the control input $u$ in (1) is not necessarily given by a static law but that it can be the output of a dynamic controller. The states of the controller would then be incorporated into the vector $x$ and we would obtain a model of the form of (4)-(5). Similarly, the controller may not be implemented using zero-order-holds as any holding function is suitable as long as the problem can be modeled by (4)-(5). For instance, the model-based technique in [11] would require to introduce an additional variable $x_{s}$, the estimate of $x$ with the notation of [11], which would correspond to $\chi$ in (4).

\section{B. Emulation-based periodic event-triggered control}

In PETC, the triggering condition can only be checked periodically every $h$ units. Intuitively, to select $h$ sufficiently small should allow to still maintain the performances ensured by the original event-triggering condition in (5). Nevertheless, it may not be possible to select $h$ as small as desired because of the hardware constraints or because we want to use the hardware as little as possible to save resources. We therefore need to develop appropriate triggering conditions for this setup. Using again the impulsive formulation following [9], the closed-loop system under a PETC strategy can be defined as

$$
\begin{aligned}
\left(\begin{array}{c}
\dot{z} \\
\dot{\eta}
\end{array}\right) & =\left(\begin{array}{c}
g(z) \\
1
\end{array}\right) \\
\left(\begin{array}{c}
z\left(\tilde{t}_{k+1}^{+}\right) \\
\eta\left(\tilde{t}_{k+1}^{+}\right)
\end{array}\right) & =\left(\begin{array}{c}
b\left(z\left(\tilde{t}_{k+1}\right)\right) \\
0
\end{array}\right), \quad \forall t \in\left[\tilde{t}_{k}, \tilde{t}_{k+1}[\right.
\end{aligned}
$$

where $\eta \in \mathbb{R}_{\geq 0}$ represents the time elapsed since the last jump and its initial condition is taken to be $\eta\left(\tilde{t}_{0}\right)=0$. The jump instants of (8) are defined as

$$
\begin{aligned}
\tilde{t}_{k+1}=\tilde{t}_{k}+\min \{t>0: & \left(\tilde{\Gamma}\left(z\left(t, b\left(z\left(\tilde{t}_{k}\right)\right)\right)\right) \geq 0\right) \\
& \left.\wedge\left(\exists n \in \mathbb{Z}_{\geq 0} \quad \eta(t, 0)=n h\right)\right\}, \\
\tilde{t}_{0}=0, &
\end{aligned}
$$

where $\tilde{\Gamma}: \mathbb{R}^{n_{z}} \rightarrow \mathbb{R}$ is a function to be designed and, as before, $z\left(t, z_{0}\right)$ denotes the solution to $\dot{z}=g(z)$ at time $t \geq 0$ starting at $z_{0}$. Since the triggering condition is not checked continuously in (9), there is no guarantee that $\Gamma$ remains negative along the solutions to (8)-(9) as in the case of standard event-triggered control. Therefore, the control objectives may not be ensured.

\footnotetext{
${ }^{1}$ The set $\Omega$ is said to be forward invariant for the impulsive system (4)-(5) if $z_{0} \in \Omega$ implies that the corresponding solution $z$ with $z\left(t_{0}\right)=z_{0}$ to (4)-(5) lies in $\Omega$ for all time larger than $t_{0}$.
}

Our aim is to provide tools for the design of $h$ and $\tilde{\Gamma}$ to guarantee that $\Gamma$ remains negative along the solutions to (8)(9) up to an error which can be rendered as small as desired. In that way, the original specifications of the ETC system (4)-(5) will be approximately maintained.

\section{MAIN RESUlts}

We first explain how to select the period $h$ and the triggering condition $\tilde{\Gamma}$. We then present the analytical results.

\section{A. Design}

Under the PETC strategy, the input can only be updated whenever the triggering condition is evaluated, that is, every $h$ units of time. Hence, it is reasonable to select the sampling period to be less than the minimum inter-transmission time of the event-triggered controlled system (4)-(5) (which does exist in view of Assumption 1). In that way, after a jump, we know that $\Gamma$ will remain strictly negative at least until the next sampling instant. Therefore, we select $h$ such that

$$
0<h<T
$$

where $T$ is defined in (7). Estimates of $T$ are generally given in the analysis of the event-triggered controlled system (4) to prove the existence of a dwell-time between two transmissions. They can also be numerically evaluated.

Since our objective is to guarantee that $\Gamma$ remains negative along the solutions to (8)-(9) up to an adjustable error, we would like to verify at $t=n h, n \in \mathbb{Z}_{>0}$, whether the condition $\Gamma(z(t)) \geq 0$ may be satisfied for $t \in] n h,(n+1) h]$. To address this question, we decompose the interval $] n h,(n+$ 1)h] into $N$ intervals of length $\frac{h}{N}$, i.e. $\left.] n h,(n+1) h\right]=$ ]$\left.n h, n h+\frac{h}{N}\right] \cup\left[n h+\frac{h}{N}, n h+2 \frac{h}{N}\right] \cup \ldots \cup[n h+(N-$ $\left.1) \frac{h}{N},(n+1) h\right]$. We then investigate whether $\Gamma(z(t))$ may become non-negative at the time instants $t=n h+i \frac{h}{N}$ for $i \in\{1,2, \ldots, N\}$. Should the condition be violated at some $t=n h+i \frac{h}{N}$ (without updating the control action), a jump must occur when $t=n h$ in order to guarantee $\Gamma\left(z\left(n h+i \frac{h}{N}\right)\right) \leq 0$ for all $i \in\{1,2, \ldots, N\}$. Obviously, $\Gamma(z(t))$ may still become positive between these time instants but we will see that the induced error will be of the order of $\frac{h}{N}$ which can be reduced by increasing $N$. Furthermore, the event-triggered control system in (4)-(5) is often tolerant to small delays (see [19], [22], [23] to mention a few). Hence, it is reasonable to allow the condition (6) to be violated for a small amount of time as it may still ensure satisfactory properties.

To determine at time $t=n h, n \in \mathbb{Z}_{>0}$, whether the condition $\Gamma\left(z\left(n h+i \frac{h}{N}\right)\right)<0, i \in\{1, \ldots, N\}$, may be violated, the evolution of the triggering function $\Gamma$ along the solutions to $\dot{z}=g(z)$ needs to be analyzed. This point is addressed by resorting to the techniques of [2] to estimate isochronous manifolds. Like in [2], we make the following assumption.

Assumption 2: The functions $g$ and $\Gamma$ are $p$-times continuously differentiable where $p \in \mathbb{Z}_{>0}$ and there exist $c, \varsigma_{j} \in \mathbb{R}$ 
for $j \in\{0,1, \ldots, p-1\}$ such that the following holds for any $z$ in $\Omega$

$$
\mathcal{L}_{g}^{p} \Gamma(z) \leq \sum_{j=0}^{p-1} \varsigma_{j} \mathcal{L}_{g}^{j} \Gamma(z)+c,
$$

where we have denoted the $j$ th Lie derivative of $\Gamma$ along the closed-loop dynamics $g$ as $\mathcal{L}_{g}^{j}$, with $\mathcal{L}_{g}^{0} \Gamma=g,\left(\mathcal{L}_{g} \Gamma\right)(z)=$ $\frac{\partial \Gamma}{\partial z} g(z)$ and $\mathcal{L}_{g}^{j} \Gamma=\mathcal{L}_{g}\left(\mathcal{L}_{g}^{j-1} \Gamma\right)$.

Note that the condition (11) differs from (V.11) in [2] due to the constant $c$. This allows us to work with a relaxed condition which applies to a larger class of systems ${ }^{2}$. Indeed, Assumption 2 always holds (when $g$ and $\Gamma$ are $p$-times continuously differentiable) as it suffices to take $c=\max _{z \in \Omega} \mathcal{L}_{g}^{p} \Gamma(z)$ and $\varsigma_{j}=0$ for $j \in\{0,1, \ldots, p-1\}$ to ensure (11) (recall that $\Omega$ is compact in view of Assumption 1). Clearly, this particular choice may lead to conservative results as we will explain. We use the property (11) in the following to estimate the set of states for which $\Gamma$ becomes positive in $i \frac{h}{N}$ units of time for system (4)-(5), which we denote $\mathcal{S}\left(i \frac{h}{N}\right):=$ $\left\{z_{0} \in \Omega:\left(\Gamma\left(z_{0}\right)<0\right)\right.$ and $\left.\left(\Gamma\left(z\left(i \frac{h}{N}, z_{0}\right)\right) \geq 0\right)\right\}, i \in$ $\{1, \ldots, N\}$. The parameter $p$ may be increased to derive tighter upper-bounds of $\mathcal{L}_{g}^{p} \Gamma(z)$ in (11) and thus tighter estimates of $\mathcal{S}\left(i \frac{h}{N}\right)$ at the price of a higher computational complexity.

Remark 2: The self-triggering formulas in [2] tend to provide accurate estimates of the time instants when $\Gamma$ becomes non-negative provided the bound (11) is tight, which may be difficult to achieve in practice. In the proposed PETC approach, the evolution of $\Gamma$ is investigated over shorter horizons than in STC, namely time-intervals of length $h$. Hence, the bound in (11) does not necessarily need to be accurate to provide satisfactory results as we expect these estimates to be tighter whenever times are shorter based on previous experience [2].

Assumption 2 allows to bound the evolution of $\Gamma$ by a linear differential equation for which the analytical solution can be computed as stated in the lemma below which directly follows from Lemma V.2 in [2].

Lemma 1: Under Assumption 2, for all solutions $z$ to $\dot{z}=$ $g(z)$ with initial condition $z_{0} \in \Omega$ such that $z\left(t, z_{0}\right) \in \Omega$ for any $t \in[0, h], \Gamma\left(z\left(t, z_{0}\right)\right) \leq y_{1}\left(t, y_{0}\right)$ for any $t \in[0, h]$, where $y_{1}$ is the first component of the solution of the linear differential equation below

$$
\left\{\begin{aligned}
\dot{y}_{j} & =y_{j+1}, \quad j \in\{1,2, \ldots, p-1\} \\
\dot{y}_{p} & =\sum_{j=0}^{p-1} \varsigma_{j} y_{j+1}+y_{p+1} \\
\dot{y}_{p+1} & =0
\end{aligned}\right.
$$

with $y_{0}=\left(\Gamma\left(z_{0}\right), \mathcal{L}_{g} \Gamma\left(z_{0}\right), \ldots, \mathcal{L}_{g}^{p-1} \Gamma\left(z_{0}\right), c\right)$.

In that way, for a given state $z_{0} \in \Omega$, if $y_{1}\left(i \frac{h}{N}, y_{0}\right)$ is positive for some $i \in\{1,2, \ldots, N\}$, then Lemma 1 implies that $\Gamma\left(z\left(i \frac{h}{N}, z_{0}\right)\right)$ may be positive. On the other hand, if $y_{1}\left(i \frac{h}{N}, y_{0}\right)$ is negative, Lemma 1 ensures that $\Gamma\left(z\left(i \frac{h}{N}, z_{0}\right)\right)$

\footnotetext{
${ }^{2}$ It is possible to derive self-triggering rules when (11) holds with $c \neq 0$ by following the approach of [2]. Nevertheless, the next transmission time will generally be given by an implicit formula in this case which may be difficult to solve on-line.
}

is negative. The term $y_{1}\left(i \frac{h}{N}, y_{0}\right)$ can be computed off-line by noting that $y\left(i \frac{h}{N}, y_{0}\right)=\mathcal{R}\left(i \frac{h}{N}, z_{0}\right)$ where

$$
\begin{aligned}
\mathcal{R}\left(i \frac{h}{N}, z_{0}\right):=\left(\begin{array}{lllll}
1 & 0 & \ldots & 0
\end{array}\right) \exp \left(A_{p} i \frac{h}{N}\right)\left(\begin{array}{c}
\Gamma\left(z_{0}\right) \\
\mathcal{L}_{g} \Gamma\left(z_{0}\right) \\
\vdots \\
\mathcal{L}_{g}^{p-1} \Gamma\left(z_{0}\right) \\
c
\end{array}\right) \\
\text { with } A_{p}:=\left(\begin{array}{ccccccc}
0 & 1 & 0 & \ldots & 0 & 0 & 0 \\
0 & 0 & 1 & \ldots & 0 & 0 & 0 \\
\vdots & & \ddots & & \vdots & \vdots \\
0 & 0 & 0 & \ldots & 1 & 0 & 0 \\
0 & 0 & 0 & \ldots & 0 & 1 & 0 \\
\varsigma_{0} & \varsigma_{1} & \varsigma_{2} & \ldots & \varsigma_{p-2} \\
0 & \varsigma_{p-1} & 0 & \ldots & 0 & 0 & 0
\end{array}\right) .
\end{aligned}
$$

Hence, we define $\tilde{\Gamma}(z)$ for any $z \in \Omega$ as

$$
\tilde{\Gamma}(z):=\max \left\{\mathcal{R}\left(i \frac{h}{N}, z\right) \mid i \in\{1, \ldots, N\}\right\} .
$$

Every $h$ units of time the current state $z$ is measured and we verify whether $\tilde{\Gamma}(z)$ is positive in which case the control input is updated. Conversely, if $\tilde{\Gamma}(z)$ is negative, then the control input is not updated. Although inspired by the selftriggering techniques, we do not propose a self-triggered emulation of the event-triggered strategy. Indeed, we do not compute the next time at which a jump should occur, but we simply verify at each sampling instant whether the state of system (8) lies in the set $\{z: \tilde{\Gamma}(z) \geq 0\}$. Note that this set can be computed off-line in view of (13) and (14). It also has to be noticed that when $\frac{T}{h} \geq 2$, we do not need to verify the triggering condition for the next $\left\lfloor\frac{T}{h}\right\rfloor-1$ sample times following a control input update according to Assumption 1, which allows to further reduce computations.

Before presenting the analytical guarantees, we need to assume that the solutions to $\dot{z}=g(z)$ which start in $\Omega$ remain in this set for the next $h$ units of times. The condition is linked to the robustness of system (4)-(5) to delays and is reasonable as we have already mentioned in this section that ETC systems are usually tolerant to delays.

Assumption 3: Consider the system $\dot{z}=g(z)$. For any $z_{0} \in \Omega, z\left(t, z_{0}\right) \in \Omega$ for all $t \in[0, h]$.

\section{B. Analytical result}

The theorem below states that to choose $h$ such that (10) holds and $\tilde{\Gamma}$ as in (14) ensures that $\Gamma$ will be negative along the solutions to (8)-(9) up to an error which can be rendered arbitrarily small by increasing $N$. The proof is given in the appendix.

Theorem 1: Consider system (8)-(9) with $h$ which satisfies (10) and $\tilde{\Gamma}$ defined in (14) and suppose Assumptions 1-3 hold. Then for any solution $z$ to (8)-(9) which is initialized in $\Omega$, the following hold.

(i) $\Gamma\left(z\left(i \frac{h}{N}\right)\right)<0$ for any $i \in \mathbb{Z}_{>0}$.

(ii) There exists $M \geq 0$ which is independent of $(h, N)$ such that $\Gamma(z(t)) \leq \frac{h}{N} M$ for any $t>0$.

Theorem 1 shows that, for a given $h$, the parameter $N$ can be increased to reduce the error induced by the sampling, which corresponds to the term $\frac{h}{N} M$ in item (ii) of Theorem 1. On the other hand, to increase $N$ leads to more 
computations since $N$ conditions need to be evaluated at each sampling instant in view of (14). It is interesting to note that constant ratios $\frac{h}{N}$ lead to the same $\tilde{\Gamma}$ and thus the same performance guarantees in view of item (ii) of Theorem 1. For a given performance specification, we are therefore free to select both $h$ and $N$ small or large. The former would typically lead to less control input updates and frequent but simple checks of the triggering condition, while the latter would generate more complex but less frequent checks and we may expect that the control input will be more often transmitted to the controller. This degree of freedom allows the designer to adapt the PETC strategy according to the setup constraints.

Remark 3: When the requirement that $\Gamma$ remains negative along the solutions to (8)-(9) for any positive time is essential, it is possible to modify the strategy proposed above by adding a term to (13) which upper-bounds the intersample behaviour of $y_{1}$ between $i \frac{h}{N}$ and $(i+1) \frac{h}{N}$ using the techniques of [7] for instance.

\section{ILlustratiVE EXAMPLE}

We apply the results of Section III to a rigid body previously studied in [2]. The model is (see [5]) $\dot{x}_{1}=u_{1}$, $\dot{x}_{2}=u_{2}, \dot{x}_{3}=x_{1} x_{2}$, and we consider the controller synthesized in [5] in order to stabilize the origin: $u_{1}=$ $-x_{1} x_{2}-2 x_{2} x_{3}-x_{1}-x_{3}$ and $u_{2}=2 x_{1} x_{2} x_{3}+3 x_{3}^{2}-x_{2}$. The implementation of the controller on a digital platform leads to the following closed-loop system

$$
\begin{aligned}
\dot{x}_{1}= & -\left(x_{1}+e_{1}\right)\left(x_{2}+e_{2}\right)-2\left(x_{2}+e_{2}\right)\left(x_{3}+e_{3}\right) \\
& -\left(x_{1}+e_{1}\right)-\left(x_{3}+e_{3}\right) \\
\dot{x}_{2}= & 2\left(x_{1}+e_{1}\right)\left(x_{2}+e_{2}\right)\left(x_{3}+e_{3}\right)+3\left(x_{3}+e_{3}\right)^{2} \\
& -\left(x_{2}+e_{2}\right) \\
\dot{x}_{3}= & x_{1} x_{2},
\end{aligned}
$$

where $e_{i}(t)=x_{i}\left(t_{k}\right)-x_{i}(t)$ for $t \in\left[t_{k}, t_{k+1}\left[\right.\right.$, with $t_{k}, k \in$ $\mathbb{Z}_{\geq 0}$, the sequence of transmissions and $i \in\{1,2,3\}$, as in Section II-A. In order to stabilize the origin of (15), we take the triggering condition as in [1], [2] which is obtained using the Lyapunov function $V(x):=\frac{1}{2}\left(x_{1}+x_{3}\right)^{2}+\frac{1}{2}\left(x_{2}-x_{3}^{2}\right)^{2}+$ $x_{3}^{2}: \Gamma(x, e):=|e|^{2}-0.79^{2} \sigma^{2}|x|^{2}$, where $x=\left(x_{1}, x_{2}, x_{3}\right)$, $e=\left(e_{1}, e_{2}, e_{3}\right)$ and $\sigma=0.8$.

We compare the amount of transmissions respectively generated by the event-triggered controller, the corresponding self-triggered controller in [2] and a periodic event-triggered controller designed by following the procedure in Section III. Assumption 1 is satisfied with $T=0.08$ (which has been numerically computed) for $\Omega=\{(x, e): V(x) \leq$ 5 and $\Gamma(x, e) \leq 0\}$ and Assumption 3 also holds. Regarding Assumption 2, we note that the system vector fields and the triggering condition are smooth. In addition, (11) is verified with $^{3} p=3, \varsigma_{0}=-748.4986, \varsigma_{1}=-1.0008, \varsigma_{2}=4.3166$ and $c=0$. We can thus apply the method presented in Section III as all the conditions of Theorem 1 are ensured. We have selected $N=1$ and $h<T$. In that way, the expression of $\tilde{\Gamma}$ in (14) reduces to $\tilde{\Gamma}(z)=\mathcal{R}(h, z)$ where $\mathcal{R}$

\footnotetext{
${ }^{3}$ SOSTools ([17]) was used to compute $\varsigma_{i}$ and $\chi_{i}$ for $i \in\{1,2,3\}$.
}

\begin{tabular}{cccc} 
ETC & \multicolumn{2}{c}{ PETC } & STC \\
& $h=0.01$ & $h=0.02$ & \\
\hline 0.3488 & 0.3440 & 0.3376 & 0.3151 \\
\hline
\end{tabular}

TABLE I

AVERAGE INTER-TRANSMISSION TIMES FOR 100 POINTS.

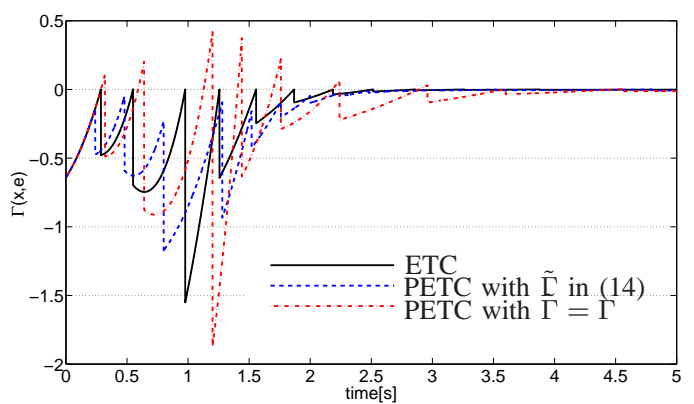

Fig. 1. Evolution of $\Gamma$ for the periodic event-triggered controlled system (15) with different choices for $\tilde{\Gamma}$.

defined in (13) with the coefficients given above. The selftriggered controller is designed as in Section VI.B in [2] where system (15) was augmented to be homogeneous. In that way, the transmission times are defined by the formula in (V.30) in [2] with ${ }^{3} p=3, \chi_{0}=-73.2528, \chi_{1}=1.7157$, $\chi_{2}=1.8299$ and $t_{*}=0.2$. Table I provides the average intertransmission times for 100 points in $\Omega$ whose $x$-components are equally spaced along the sphere centered at 0 and of radius 1 and whose $e$-components are set to 0 . PETC generates inter-transmission times which are smaller than in ETC but larger than in STC. Moreover, we expect the average inter-transmission time to increase when the sampling period $h$ decreases as suggested by Table I.

One might wonder the interest of designing a specific function $\tilde{\Gamma}$ to derive a periodic event-triggered controller compared to the case where we simply take $\tilde{\Gamma}=\Gamma$, in particular when $N=1$. Indeed, it can be noted that to take $\tilde{\Gamma}=\Gamma$ also ensures the item (ii) of Theorem 1 by following similar lines as in the proof of Theorem 1, but it does not guarantee the item (i) of Theorem 1. We plotted for that purpose the evolution of $\Gamma$ for these two options in Figure 1 with $h=0.079$ and the same initial conditions. We see that $\Gamma$ remains negative all the time with $\tilde{\Gamma}$ given by (14), which implies that the periodic event-triggered controller ensures the same specification as the event-triggered controller, while $\Gamma$ often reaches positive values when $\tilde{\Gamma}=\Gamma$.

On the other hand, we would expect PETC to be more robust to measurement noises, external disturbances and model uncertainties than STC. Indeed, the state is periodically monitored in PETC and the evolution of $\Gamma$ is only predicted in some sense on a horizon of length $h$, while the STC method of [2] considers the evolution of $\Gamma$ on longer time intervals. To illustrate these points, we have added a constant external additive disturbance to each equation in (15) from $t=0.5$ to $t=1$. The disturbance value was set to 12 and we have run 100 simulations with the same initial conditions as for Table I. The simulations have shown that PETC with $h=0.01$ asymptotically stabilizes the origin for $91 \%$ of the simulations while STC only does it for $3 \%$. 
These observations confirm the expected robustness features of PETC compared to STC.

\section{CONCLUSiOns}

A method for the design of periodic event-triggered controllers for nonlinear systems has been presented. The approach consists in emulating a known event-triggered controller. It has been explained how the sampling period should be selected and how the triggering condition needs to be redesigned to approximately ensure the same performances as in ETC. In that way, this study offers guidelines for the implementation of event-triggered controllers in practice. It would be interesting in the future to analytically investigate the robustness properties of the proposed PETC strategies. On the other hand, we have focused on the case where an event-triggered controller has been a priori designed. In [8], [9], the problem is tackled from a different point of view: the periodic event-triggered controller is derived from a periodically sampled controller. Analogous results for nonlinear systems would be very appealing though challenging.

\section{APPENDIX}

Proof of Theorem 1. Let $z_{0} \in \Omega$, we consider the solution $(z, \eta)$ to (8)-(9) with initial condition $z\left(t_{0}\right)=z_{0}, \eta\left(t_{0}\right)=0$ and initial time $t_{0}=0$. Denote $t_{\max } \in \mathbb{R}_{\geq 0} \cup\{\infty\}$ the maximum existence time of $(z, \eta)$. Let $\tilde{z}$ be the solution to (4)-(5) with initial condition $\tilde{z}\left(t_{0}\right)=z_{0}$. Note that $\tilde{z}$ is defined for all positive time in view of Section II-A. According to (5) and (9), both $z$ and $\tilde{z}$ jump at $t=0$. Hence $\tilde{z}$ and $z$ remain equal until one of the two solutions jumps again since they have the same dynamics during flow. We know that $z$ will not jump before $t=h$, neither will $\tilde{z}$ on the interval $] 0, h]$ since $h<T$ where $T$ is the minimum inter-transmission time (see Assumption 1). Hence, since $z$ and $\tilde{z}$ are left-continuous, $z(t)=\tilde{z}(t)$ for any $t \in] 0, h]$ from which we deduce that $t_{\max } \geq h, \Gamma(z(t))=\Gamma(\tilde{z}(t))<0$, and $\tilde{z}(t) \in \Omega$ for any $t \in] 0, h]$ as $\Omega$ is forward invariant for system (4)-(5) by virtue of Assumption 1. Consider $t=h$. If $\tilde{\Gamma}(z(h)) \geq 0$, then a jump occurs and we follow similar arguments as above to conclude that $t_{\max } \geq 2 h$ and that $\Gamma(z(t))<0$ for all $t \in] h, 2 h]$. Suppose now that $\tilde{\Gamma}(z(h))<$ 0 . No jump occurs for system (8)-(9). Consequently, $z(t)$ remains in $\Omega$ for $t \in[h, 2 h]$ in view of Assumption 3. We deduce from Lemma 1 and the definition of $\tilde{\Gamma}$ in (14) that

$$
\Gamma\left(z\left(h+i \frac{h}{N}\right)\right)<0 \quad \forall i \in\{1,2, \ldots, N-1\},
$$

as $\tilde{\Gamma}(z(h))<0$. Suppose there exists $t \in] h+i \frac{h}{N}, h+(i+$ 1) $\frac{h}{N}$ [ for some $i \in\{0,1, \ldots, N-1\}$ such that $\Gamma(z(t)) \geq$ 0 . Using the mean value theorem (since $t \mapsto \Gamma(z(t))$ is continuously differentiable on $[0, h]$ in view of Assumption 2 and (8)), we derive that $\Gamma(z(t))=\Gamma\left(z\left(h+i \frac{h}{N}\right)\right)+(t-$ $\left.h-i \frac{h}{N}\right) \mathcal{L}_{g} \Gamma\left(z\left(t^{*}\right)\right)$ with $t^{*} \in\left[h+i \frac{h}{N}, t\right]$. Hence, $\Gamma(z(t)) \leq$ $\Gamma\left(z\left(h+i \frac{h}{N}\right)\right)+\left|t-h-i \frac{h}{N}\right|\left|\mathcal{L}_{g} \Gamma\left(z\left(t^{*}\right)\right)\right|$. Since $\left.t \in\right] i \frac{h}{N},(i+$ 1) $\frac{h}{N}\left[, \Gamma(z(t)) \leq \Gamma\left(z\left(h+i \frac{h}{N}\right)\right)+\frac{h}{N}\left|\mathcal{L}_{g} \Gamma\left(z\left(t^{*}\right)\right)\right|\right.$. Using the fact that $z\left(t^{*}\right) \in \Omega$, we define $M=\max _{y \in \Omega}\left\{\left|\mathcal{L}_{g} \Gamma(y)\right|\right\}$ which is well-defined since $\Omega$ is compact and $\mathcal{L}_{g} \Gamma$ is continuous in view of Assumptions 1 and 2, respectively. We note that
$M$ is independent of $h$ and $N$. We derive that $\Gamma(z(t)) \leq$ $\Gamma\left(z\left(h+i \frac{h}{N}\right)\right)+\frac{h}{N} M$. Using that $\Gamma\left(z\left(h+i \frac{h}{N}\right)\right)<0$ in view of (16), we obtain $\Gamma(z(t)) \leq \frac{h}{N} M$. Hence for any $t \in[h, 2 h]$, it holds that $\Gamma(z(t)) \leq \frac{h}{N} M$. The desired properties are ensured by induction.

\section{REFERENCES}

[1] A. Anta and P. Tabuada. To sample or not to sample: self-triggered control for nonlinear systems. IEEE Transactions on Automatic Control, 55(9):2030-2042, 2010.

[2] A. Anta and P. Tabuada. Exploiting isochrony in self-triggered control. IEEE Trans. on Automatic Control, 57(4):950-962, 2012.

[3] K.E. Arzén. A simple event-based PID controller. In $14^{\text {th }}$ IFAC World Congress, Beijing, China, pages 423-428, 1999.

[4] K.J. Aström and B.M. Bernhardsson. Comparison of periodic and event based sampling for first-order stochastic systems. In Proceedings of the 14th IFAC World congress, volume 11, pages 301-306, 1999.

[5] C.I. Byrnes and A. Isidori. New results and examples in nonlinear feedback stabilization. Syst. \& Control Letters, 12(5):437-442, 1989.

[6] M.C.F. Donkers and W.P.M.H. Heemels. Output-based event-triggered control with guaranteed $\mathcal{L}_{\infty}$-gain and improved and decentralized event-triggering. IEEE Trans. on Automatic Control, 57(6):1362-1376, 2012.

[7] M.C.F. Donkers, W.P.M.H. Heemels, N. van de Wouw, and L. Hetel. Stability analysis of networked control systems using a switched linear systems approach. IEEE Transactions on Automatic Control, 56(9):2101-2115, 2011.

[8] W.P.M.H. Heemels and M.C.F. Donkers. Model-based periodic eventtriggered control for linear systems. Automatica, 49(3):698-711, 2013.

[9] W.P.M.H. Heemels, M.C.F. Donkers, and A.R. Teel. Periodic eventtriggered control for linear systems. IEEE Transactions Automatic Control, 58(4):847-861, 2013.

[10] W.P.M.H. Heemels, J.H. Sandee, and P.P.J. van den Bosch. Analysis of event-driven controllers for linear systems. International Journal of Control, 81(4):571-590, 2009.

[11] J. Lunze and D. Lehmann. A state-feedback approach to event-based control. Automatica, 46:211-215, 2010.

[12] M. Mazo Jr. and P. Tabuada. Decentralized event-triggered control over wireless sensor/actuator networks. In IEEE Transactions on Automatic Control, Special Issue on Wireless Sensor and Actuator Networks, volume 56, pages 2456-2461, 2011.

[13] M. Miskowicz. Send-on-delta concept: An event-based data reporting strategy. Sensors, 6(1):49-63, 2006.

[14] G. Otanez, J.R. Moyne, and D.M. Tilbury. Using deadbands to reduce communication in networked control systems. In American Control Conference, pages 3015-3020, 2002.

[15] R. Postoyan, A. Anta, D. Nešić, and P. Tabuada. A unifying Lyapunovbased framework for the event-triggered control of nonlinear systems. In IEEE Conference on Decision and Control and European Control Conference, Orlando, U.S.A., pages 2559-2564, 2011.

[16] R. Postoyan, P. Tabuada, D. Nešić, and A. Anta. Event-triggered and self-triggered stabilization of networked control systems. In IEEE Conference on Decision and Control and European Control Conference, Orlando, U.S.A., pages 2565-2570, 2011.

[17] S. Prajna, A. Papachristodoulou, P. Seiler, and P.A. Parrilo. Sostools: sum of squares optimization toolbox for matlab. URL: http://www.cds.caltech.edu/sostools, 2004.

[18] R.G. Sanfelice and A.R. Teel. Lyapunov analysis of sampled-and-hold hybrid feedbacks. In IEEE Conference on Decision and Control, San Diego, U.S.A., pages 4879-4884, 2006.

[19] P. Tabuada. Event-triggered real-time scheduling of stabilizing control tasks. IEEE Trans. on Automatic Control, 52(9):1680-1685, 2007.

[20] M. Velasco, J. Fuertes, and P. Marti. The self triggered task model for real-time control systems. 24th IEEE Real-Time Systems Symposium, pages $67-70,2003$.

[21] X. Wang and M.D. Lemmon. Self-triggered feedback control systems with finite-gain $\mathcal{L}_{2}$ stability. IEEE Transactions on Automatic Control, 45:452-467, 2009.

[22] X. Wang and M.D. Lemmon. Event-triggering in distributed networked control systems. IEEE Trans. on Aut. Control, 56(3):586-601, 2011.

[23] X. Wang and M.D. Lemmon. On event design in event-triggered feedback systems. Automatica, 47(10):2319-2322, 2011. 